\title{
Avaliação clínica da descompressão medular anterior em portadores de mielopatia cervical
}

\author{
Rodrigo Rezende1, Fernando Junqueira de Faria², \\ Igor Machado Cardoso ${ }^{3}$, José Lucas Batista Junior³, Chárbel Jacob Júnior³ \\ Hospital Santa Casa de Misericórdia de Vitória, Vitória, ES, Brasil.
}

\section{RESUMO}

Objetivo: Avaliação clínica neurológica pré e pós-operatória de pacientes portadores de mielopatia cervical submetidos à descompressão medular por via anterior. Métodos: Utilização das escalas de Nurick e de JOA (Japanese Orthopaedic Association), adaptadas para a população brasileira, no período pré e pós-operatório, como método de mensuração do grau de comprometimento neurológico, aplicando-se o teste $t$ de Student e o teste de Wilcoxon, com significância $p<0,05$. Resultados: Participaram do estudo 30 pacientes. A média do JOA no pré-operatório foi de $9,5(D P=2,8)$ e no pós-operatório, de 13,5 $(D P=3,0)$. O valor médio de Nurick foi de 3,7 $(D P=1,2)$ e 2,1 $(D P=1,2)$ no pré e pós-operatório, respectivamente. Conclusão: $A$ descompressão medular via anterior melhorou em cerca de $40 \%$ o quadro neurológico inicial, portanto pode ser considerada uma real opção de tratamento para pacientes portadores de mielopatia cervical.

\section{PALAVRAS-CHAVE}

Doenças da medula espinal, descompressão cirúrgica, avaliação de resultados.

\section{ABSTRACT}

Clinical evaluation of anterior spinal cord decompression in cervical myelopathy

Objective: Clinical neurological evaluation of preoperative and postoperative patients with cervical myelopathy who underwent anterior spinal decompression through. Methods: Use scales of Nurick and JOA (Japanese Orthopaedic Association), adapted for the Brazilian population, in the pre and postsurgery as a method of measuring the degree of neurological impairment by applying the Student $t$ and Wilcoxon test with significance $\mathrm{p}<0,05$. Results: The study included 30 patients. The mean preoperative $J O A$ was $9,5(S D=2,8)$ and postoperative $13,5(S D=3,0)$. The average Nurick was 3,7 $(S D=1,2)$ and $2,1(S D=1,2)$ before and after surgery respectively. Conclusion: It was concluded that the anterior spinal decompression improved by about $40 \%$ early neurological status, therefore can be considered a real treatment option for patients with myelopathy.

\section{KEYWORDS}

Spinal cord diseases, decompression surgical, outcome assessment.

1 Ortopedista e cirurgião da coluna, chefe do Grupo de Coluna Vertebral do Hospital Santa Casa de Misericórdia de Vitória, Vitória, ES, Brasil, e do Vila Velha Hospital, Vila Velha, ES, Brasil.

2 Graduando do curso de Medicina da Escola Superior de Ciências da Saúde da Santa Casa de Misericórdia (Emescam), Vitória, ES, Brasil.

3 Ortopedista, especialista em coluna, médico-assistente do Grupo de Coluna Vertebral do Hospital Santa Casa de Misericórdia de Vitória, Vitória, ES, Brasil. 


\section{Introdução}

A mielopatia cervical espondilolítica é uma disfunção da medula espinhal relacionada à degeneração típica do envelhecimento, cuja patologia primária se relaciona a uma compressão da medula espinhal cervical. Acomete principalmente pacientes do sexo masculino entre a quinta e a sétima década de vida. As manifestações clínicas dessa doença cursam com alterações do moto neurônio superior, caracterizadas por hiper-reflexia e distúrbios da marcha e da coordenação. ${ }^{1-3}$

São frequentes as alterações dos movimentos finos da extremidade superior, fadiga precoce aos movimentos repetitivos e perda da coordenação motora. O distúrbio da marcha é uma das primeiras manifestações clínicas da mielopatia cervical, que ocorre de forma lenta e gradual, muitas vezes passando despercebido pelo paciente, que sente, inicialmente, desequilíbrio e falta de coordenação para fazer curvas. A perda do controle vesical e intestinal pode ocorrer em $20 \%$ a $50 \%$ dos pacientes, porém é uma manifestação tardia da doença. ${ }^{4-6}$

O tratamento cirúrgico da mielopatia cervical secundária à estenose de canal ainda permanece de difícil resolução, pois existem dúvidas sobre qual a melhor técnica cirúrgica a ser utilizada. Outra dúvida existente ocorre nos casos de pacientes com comprometimento neurológico grave, em que se questionam se a cirurgia de descompressão medular pode causar alguma melhora clínica representativa na qualidade de vida desses pacientes. Apesar de existirem diversos critérios pré-operatórios, como o número de níveis acometidos, angulação cervical e localização da compressão, não existe uma definição na literatura sobre qual a melhor técnica de descompressão.

Muitos autores preconizam a descompressão por via anterior por ser de mais fácil acesso e também por ser possível realizar discectomia associada ou não à corpectomia; outros preconizam a descompressão por via posterior principalmente nos casos em que a angulação cervical for lordótica e existirem mais de três níveis de compressão. Mais recentemente, alguns autores têm optado pela descompressão 360 graus, ou seja, descompressão anterior e posterior. Essa conduta mais agressiva se deve ao fato de, muitas vezes, o fator causador da mielopatia estar localizado na região anterior, devido a uma hérnia discal ou a fatores mecânicos como a inversão da lordose cervical e também devido a hipertrofia facetária e do ligamento amarelo na região posterior, devendo ambas as regiões serem descomprimidas.

Embora haja diversas técnicas de descompressão, nenhuma é consenso entre os cirurgiões. Acreditamos que a descompressão medular por via anterior proporciona adequada descompressão medular, com consequente melhora clínica dos pacientes portadores de mielopatia cervical, podendo, dessa forma, por meio de uma única cirurgia, se realizar a descompressão adequada, evitando-se muitas vezes outros procedimentos cirúrgicos, diminuindo-se, assim, a morbidade de uma descompressão posterior ou 360 graus.

Na literatura são encontrados poucos trabalhos que avaliam clinicamente os resultados da descompressão medular em pacientes portadores de mielopatia.

Este trabalho foi desenvolvido com o objetivo de avaliar clinicamente, por meio das escalas de JOA ( $\mathrm{Ja}$ panese Orthopaedic Association) e Nurick, os resultados da descompressão medular por via anterior de pacientes portadores de mielopatia cervical.

\section{Material e métodos}

Estudo prospectivo, de caráter descritivo e comparativo, no qual foram avaliados 30 pacientes portadores de mielopatia cervical, em período pré e pós-operatório, de descompressão medular via anterior.

Entre os 30 pacientes avaliados, seis eram do sexo feminino, com idade média de 56,1 anos, variando de 41 a 67 anos, e 24 eram do sexo masculino, com idade média de 51,7 anos, variando de 26 a 73 anos.

O presente estudo foi iniciado a partir de uma autorização, por escrito, emitida pelo Comitê de Ética em Pesquisa com Seres Humanos da Escola Superior de Ciências da Santa Casa de Misericórdia de Vitória (Emescam).

Os pacientes foram convidados a participar do estudo, mediante os esclarecimentos necessários, por meio do Termo de Consentimento Livre e Esclarecido, o qual deveria ser assinado em caso de concordância, para que pudessem ser incluídos na pesquisa.

Utilizaram-se as escalas de JOA e de Nurick para avaliar o quadro neurológico dos pacientes. A escala de JOA (Anexo 1) avalia o grau de disfunção motora da extremidade superior e inferior, assim como a disfunção sensitiva e a esfincteriana. Por meio dela é possível graduar numericamente o grau de comprometimento clínico causado pela doença, sendo considerados normais pacientes com resultados entre $12 \mathrm{e} 17$ pontos. Em valores abaixo de 12 pontos, o paciente é considerado portador de mielopatia cervial. ${ }^{7-10}$

Já a escala de Nurick (Anexo 2) analisa principalmente o grau de independência para deambulação. Sua graduação varia do grau 0 - grau em que o paciente apresenta sinais de comprometimento radicular, mas sem evidências de comprometimento medular - até o grau 5 - em que o paciente se torna incapaz de deambular, estando restrito ao leito ou à cadeira de rodas. Dessa forma, quanto maior for a graduação do paciente, mais grave será seu déficit motor. ${ }^{9,11}$ 
Como critério de inclusão do presente estudo, foram utilizados pacientes portadores de mielopatia de caráter degenerativo, no período pré-operatório, com documentação pela ressonância magnética apresentando sinais de mielopatia cervical, com compressão máxima de até três níveis e que apresentavam protocolos de avaliação clínica, escalas de JOA e Nurick preenchidas pré e pós-operatoriamente.

Foram excluídos do estudo os pacientes com mielopatia cervical secundária a processos infecciosos, tumorais, doenças metabólicas e trauma, assim como pacientes com compressão em mais de três níveis cervicais.

O estudo comparativo foi feito pela análise dos valores absolutos encontrados na avaliação clínica da JOA e do Nurick de cada paciente pré e pós-operatório.

A avaliação do resultado pós-operatório foi realizada no quarto mês posterior à cirurgia, pela mesma equipe médica, quando então foram novamente preenchidas as escalas de JOA e Nurick.

Os resultados do trabalho foram demonstrados por meio dos cálculos da média \pm desvio-padrão (DP) e dos valores de $p$, pela utilização do teste $t$ de Student não pareado e do teste de Wilcoxon, ambos com significância dada para valores de $p<0,05$.

\section{Resultados}

Dos 30 pacientes avaliados no pré-operatório, observou-se que 24 (80\%) deles apresentavam Nurick 3,4 ou 5 e que apenas $2(6,66 \%)$ deles apresentavam quadro neurológico mínimo, classificado como Nurick 1 , conforme tabela 1 , sendo o valor médio absoluto do Nurick de 3,7.

Ao serem analisados os resultados absolutos do valor médio de Nurick pré e pós-operatório, percebeu-se uma evolução média de $3,7(\mathrm{DP}=1,2)$ para $2,1(\mathrm{DP}=$ 1,2 ), respectivamente (Tabela 2), demonstrando haver melhora clínica no pós-operatório e estatisticamente significativa, $\operatorname{com} \mathrm{p}=0,000(\mathrm{p}<0,05)$.

Quando analisada especificamente cada graduação da escala de Nurick, observou-se melhora em todos os níveis, embora se destaque que quanto maior a graduação da escala de Nurick pré-operatória, percentualmente maior foi a melhora na graduação de Nurick, ou seja, pacientes que apresentavam Nurick 5 ou 4 evoluíram para melhora média de 1,75 e 2,25 pontos, respectivamente, no pós-operatório, conforme tabela 3.

Em relação aos valores absolutos de JOA pré e pós-operatórios, observou-se aumento progressivo dos resultados, e os valores médios encontrados foram de $9,5(\mathrm{DP}=2,8)$ e $13,5(\mathrm{DP}=3,0)$, respectivamente, o que demonstrou haver melhora clínica percentual de 41,65\% e estatisticamente significativa no pós-operatório, com o valor $\mathrm{p}=0,000(\mathrm{p}<0,05)$, de acordo com tabela 4 .

\begin{tabular}{lc}
\hline & $\begin{array}{c}\text { Tabela 1 - Distribuição dos pacientes pela } \\
\text { escala de Nurick no pré-operatório }\end{array}$ \\
\hline Nurick pré-operatória & Número de pacientes \\
\hline Grau 5 & 8 \\
Grau 4 & 12 \\
Grau 3 & 4 \\
Grau 2 & 4 \\
Grau 1 & 2 \\
Total & 30 \\
\hline
\end{tabular}

Ao serem analisados os resultados absolutos do valor médio de Nurick pré e pós-operatório, percebeu-se uma evolução média de $3,7(\mathrm{DP}=1,2)$ para $2,1(\mathrm{DP}=1,2)$, respectivamente (Tabela 2), demonstrando haver melhora clínica no pós-operatório e estatisticamente significativa, com $\mathrm{p}=0,000(\mathrm{p}<0,05)$.

\begin{tabular}{lccc}
\hline \multicolumn{4}{c}{ Tabela 2 - Média pré e pós-operatória da escala de Nurick } \\
\hline $\begin{array}{l}\text { Média Nurick } \\
\text { pré-operatória }\end{array}$ & $\begin{array}{c}\text { Média Nurick } \\
\text { pós-operatória }\end{array}$ & $\begin{array}{c}\text { Percentual de } \\
\text { melhora }(\%)\end{array}$ & Significância $p$ \\
\hline $3,7(\mathrm{DP}=1,2)$ & $2,1(\mathrm{DP}=1,2)$ & $41,80 \%$ & 0,000 \\
\hline
\end{tabular}

\begin{tabular}{|c|c|c|}
\hline \multicolumn{3}{|c|}{ Tabela 3 - Média de melhora pela escala de Nurick } \\
\hline Nurick pré-operatória & $\begin{array}{c}\text { Média de melhora } \\
\text { Nurick } \\
\text { pós-operatória }\end{array}$ & $\begin{array}{c}\text { Percentual de } \\
\text { melhora (\%) }\end{array}$ \\
\hline 5 & 1,75 & $35,0 \%$ \\
\hline 4 & 2,25 & $56,25 \%$ \\
\hline 3 & 0,75 & $25,0 \%$ \\
\hline 2 & 0,50 & $25,0 \%$ \\
\hline 1 & 0,0 & $0 \%$ \\
\hline
\end{tabular}

\begin{tabular}{lccc}
\hline \multicolumn{4}{c}{ Tabela 4 - Média pré e pós-operatória da escala de JOA } \\
\hline $\begin{array}{l}\text { Média do JOA } \\
\text { pré-operatória }\end{array}$ & $\begin{array}{c}\text { Média do JOA } \\
\text { pós-operatória }\end{array}$ & $\begin{array}{c}\text { Percentual de } \\
\text { melhora }(\%)\end{array}$ & Significância $p$ \\
\hline $9,5(\mathrm{DP}=2,8)$ & $13,5(\mathrm{DP}=3,0)$ & 41,65 & 0,000 \\
\hline
\end{tabular}

\section{Discussão}

A mielopatia cervical espondilolítica acomete a medula espinhal de forma progressiva e acarreta graus variados de incapacidade funcional. ${ }^{12}$ Os maus resultados cirúrgicos estão associados à gravidade do quadro neurológico, ao tempo de evolução dos sintomas e à síndrome de lesão medular central, o que reforça a necessidade de diagnóstico e tratamento precoces para a obtenção de melhor resultado clínico. ${ }^{13}$ 
A cirurgia descompressiva tem se mostrado efetiva no alívio e recuperação neurológica. Sampath et al. ${ }^{14}$ compararam pacientes tratados conservadoramente e cirurgicamente. $\mathrm{O}$ grupo tratado cirurgicamente apresentou melhora significativa dos sintomas neurológicos e da funcionalidade quando comparado ao grupo tratado conservadoramente.

Atualmente na literatura o tratamento cirúrgico para os casos de pacientes portadores de mielopatia cervical já é consenso e o que ainda não se encontra bem definido é qual a melhor técnica a ser utilizada e qual o porcentual de melhora que ocorre no pós-operatório. Vários estudos como o de Cheung et al. ${ }^{15}$ e Fessler et al. ${ }^{16}$ demonstraram que ocorreu melhora com o tratamento cirúrgico da mielopatia, porém eles não utilizaram escalas de avaliação clínica no pré- e pós-operatório e não deixaram claro qual a melhor técnica a ser utilizada. Dessa forma, permanece a dúvida de quanto é a real melhora e, consequentemente, os resultados não podem ser comparados entre as diversas escolas médicas. Às vezes a melhora clínica não corresponde à melhora na qualidade de vida desses pacientes, e esses fatos criam dúvidas sobre a efetividade do tratamento cirúrgico.

No presente trabalho realizou-se a avaliação clínica neurológica pré e pós-operatória utilizando as escalas de JOA e Nurick, que avaliam funções motoras, sensitivas e esfincterianas, e foi observado que a descompressão medular por via anterior levou a uma melhora clínica de aproximadamente $40 \%$.

Outro fato observado foi que, quanto pior o grau da mielopatia, maior a porcentagem de melhora clínica, o que demonstra valer a pena realizar a descompressão medular, independente do quadro neurológico inicial, pois muitas vezes uma pequena melhora clínica pode levar a uma grande melhora na qualidade de vida desses pacientes.

Concordamos também que muitas vezes apenas a descompressão via anterior não é suficiente e que devemos associá-la à descompressão via posterior, dando maior possibilidade de melhora ao paciente, porém novos estudos devem ser realizados com o objetivo de se avaliar a melhora clínica em pacientes submetidos à descompressão por via anterior associada à via posterior.

\section{Conclusão}

A descompressão medular realizada por via anterior em pacientes portadores de mielopatia cervical espondilolítica mostrou-se eficaz em todos os casos operados, e a melhor resposta clínica foi encontrada em pacientes com maior comprometimento neurológico.

\section{Conflito de interesses}

Os autores declaram inexistência de conflito de interesse na realização deste trabalho.

\section{Referências}

1. Baron EM, Young WF. Cervical spondylotic myelopathy: a brief review of its pathophysiology, clinical course, and diagnosis. Neurosurgery. 2007;60(Supp1 1):S35-41.

2. Tracy JA, Bartleson JD. Cervical spondylotic myelopathy. Neurologist. 2010;16(3):176-87.

3. Young WF. Cervical spondylotic myelopathy: a common cause of spinal cord dysfunction in older persons. Am Fam Physician. 2000;62(5):1064-70.

4. Yukawa $\mathrm{Y}$, Kato $\mathrm{F}$, Ito $\mathrm{K}$, Horie $\mathrm{Y}$, Nakashima $\mathrm{H}$, Masaaki $\mathrm{M}$, et al. "Ten second step test" as a new quantifiable parameter of cervical myelopathy. Spine (Phila Pa 1976). 2009;34(1):82-6.

5. Mihara $\mathrm{H}$, Kondo S, Murata A, Ishida K, Niimura T, Hachiya $M$. A new performance test for cervical myelopathy: the triangle step test. Spine (Phila Pa 1976). 2010;35(1):32-5.

6. Rhee JM, Heflin JA, Hamasaki T, Freedman B. Prevalence of physical signs in cervical myelopathy: a prospective, controlled study. Spine (Phila Pa 1976). 2009;34(9):890-5.

7. Bartels RH, Verbeek AL, Benzel EC, Fehlings MG, Guiot $\mathrm{BH}$. Validation of a translated version of the modified Japanese orthopaedic association score to assess outcomes in cervical spondylotic myelopathy: an approach to globalize outcomes assessment tools. Neurosurgery. 2010;66(5):1013-6.

8. Holly LT, Matz PG, Anderson PA, Groff MW, Heary RF, Kaiser $M G$, et al. Functional outcomes assessment for cervical degenerative disease. J Neurosurg Spine. 2009;11(2):238-44.

9. Vitzthum HE, Dalitz K. Analysis of five specific scores for cervical spondylogenic myelopathy. Eur Spine J. 2007;16(12):2096-103.

10. Nikaido T, Kikuchi S, Yabuki S, Otani K, Konno S. Surgical treatment assessment using the Japanese orthopedic association cervical myelopathy evaluation questionnaire in patients with cervical myelopathy: a new outcome measure for cervical myelopathy. Spine (Phila Pa 1976). 2009;34(23):2568-72.

11. Revanappa KK, Rajshekhar V. Comparison of Nurick grading system and modified Japanese Orthopaedic Association scoring system in evaluation of patients with cervical spondylotic myelopathy. Eur Spine J. 2011;20(9):1545-51.

12. Matz PG, Anderson PA, Holly LT, Groff MW, Heary RF, Kaiser MG, et al. The natural history of cervical spondylotic myelopathy. J Neurosurg Spine. 2009;11(2):104-11.

13. Ebersold MJ, Pare MC, Quast LM. Surgical treatment for cervical spondylitic myelopathy. J Neurosurg. 1995;82(5):745-51.

14. Sampath P, Bendebba M, Davis JD, Ducker TB. Outcome of patients treated for cervical myelopathy. A prospective, multicenter study with independent clinical review. Spine (Phila Pa 1976). 2000;25(6):670-6.

15. Cheung WY, Arvinte D, Wong YW, Luk KD, Cheung KM. Neurological recovery after surgical decompression in patients with cervical spondylotic myelopathy - A prospective study. Int Orthop. 2008;32(2):273-8. 
16. Fessler RG, Steck JC, Giovanini MA. Anterior cervical corpectomy for cervical spondylotic myelopathy. Neurosurgery. 1998;43(2):257-65.
Endereço para correspondência

Rodrigo Rezende

Serviço de Coluna Vertebral do Hospital Santa

Casa de Misericórdia de Vitória

Rua Doutor João Santos Neves, 143, Vila Rubim

29018-180 - Vitória, ES, Brasil

Telefone: (27) 3389-0305

E-mail: rezenderodrigo@hotmail.com

Anexo 1 - Escala de JOA (Japanese Orthopaedic Association)

Escala de JOA

I. Função motora de membro superior

Impossível comer com colher ou abotoar camisa

Possível comer com colher, mas impossível abotoar camisa

Possível abotoar camisa com grande dificuldade

Possível abotoar camisa com dificuldade

Normal

2

II. Função motora de membro inferior

Impossível

Necessita de bengala ou auxílio em solo plano

Necessita de auxílio em escadas

Anda sem auxílio, mas lentamente

Normal

III. Função sensitiva

Membro superior

Déficit sensitivo aparente

Déficit sensitivo mínimo

0

Normal

0

2

2

Membro inferior

Déficit sensitivo aparente

Déficit sensitivo mínimo

Normal

Tronco

Déficit sensitivo aparente

Déficit sensitivo mínimo

Normal

IV. Função vesical

Retenção urinária ou incontinência

Sensação de retenção/perda de pequeno fluxo

Retenção urinária e/ou polaciúria

Normal

0
1
2
3

Anexo 2 - Escala de Nurick

\begin{tabular}{ll}
\hline Escala de Nurick & \\
\hline Grau 0 & Sinais radiculares, sem evidências de comprometimento radicular \\
Grau 1 & Sinais de comprometimento medular com marcha normal \\
Grau 2 & Marcha comprometida, capaz de trabalhar \\
Grau 3 & Marcha comprometida, incapaz de trabalhar, mas andando seguro \\
Grau 4 & Capaz de andar com auxílio \\
Grau 5 & Cadeira de rodas ou restrito ao leito \\
\hline
\end{tabular}

